\title{
ПРЕДМЕНСТРУАЛЬНЫЙ СИНДРОМ:
} OT ТЕОРИИ K ПРАКТИКЕ

\begin{abstract}
В работе представлен обзор накопленных данных, касающихся основных дефиниций в диагностике и лечении предменструального синдрома, и собственный опыт в виде клинических случаев. Расставлены акценты на различные терапевтические подходы с точки зрения доказательной медицины.
\end{abstract}

Ключевые слова: предменструальный синдром, комбинированная оральная контрацепция, дроспиренон, этинилэстрадиол.

O.V. YAKUSHEVSKAYA, PhD in medicine, Kulakov Scientific Center of Obstetrics, Gynecology and Perinatology of the Ministry of Health of Russia PREMENSTRUAL SYNDROME: FROM THEORY TO PRACTICE

The paper presents a review of accumulated data relating to major definitions in diagnostics and therapy of the premenstrual syndrome and own experience represented by case histories. Various therapeutic approaches from the point of view of evidencebased medicine are highlighted.

Keywords: premenstrual syndrome, combined oral contraception, drospirenon, ethinyl estradiol.

редменструальный синдром (ПМС), также синдром предменструального напряжения, предменструальная болезнь, циклический синдром, сложный циклический симптомокомплекс, возникающий в предменструальные дни (за 2-12 дней до менструации) и характеризующийся различными соматическими, нейропсихическими, вегетососудистыми и обменно-эндокринными нарушениями, как правило отрицательно сказывающимися на привычном образе и качестве жизни женщин. Распространенность ПМС достигает 3-30\%, а доля предменструальных дисфорических расстройств (ПМДР) (т. е. тяжелой формы ПМС) составляет 5-8\% [1, 2].

В настоящее время точные этиологические звенья патогенеза развития ПМС остаются неизвестными. Хотя яичниковая циклическая активность, эффекты эстрадиола и прогестерона на нейротрансмиттеры остаются ключевыми факторами. Эстрогены в основном взаимодействуют с серотонинергическими, норадренергическими и опиоидными рецепторами, оказывая стимулирующее влияние. Прогестерон и его метаболиты активизируют ГАМК-эргические структуры (ГАМК - ү-аминомаслянная кислота), осуществляя седативное влияние. Причем уровни половых стероидов могут оставаться в пределах референсных значений, а их физиологические колебания в течение цикла способствуют развитию неадекватной реакции различных функциональных систем организма [3, 4]. Отсутствие ПМС до полового созревания, в период беременности и в менопаузе подтверждает вовлеченность механизма циклической яичниковой активности. Быстро меняющиеся уровни эстрадиола не только перед менструацией, но и в послеродовом и перименопаузальном периодах часто приводят к развитию гормонзависимых депрессивных расстройств [5].

Решающее значение в лечении ПМС имеет верификация правильного диагноза, которая не должна основываться на ретроспективном сборе анамнеза и жалоб пациентки. Необходимо в течение 2-3 циклов с помощью специализированных анкет-опросников выявить детально все имеющиеся жалобы у пациентки [6]. Национальная ассоциация по исследованию предменструального синдрома (National Association for Premenstrual Syndrome) разработала анкету-опросник по оценке ПМС, которая представлена на веб-сайте NAPS (www.pms.org.uk) [7]. На сегодняшний день идентифицировано более 150 симптомов ПМС. В зависимости от степени тяжести выделяют несколько вариантов ПМС (табл. 1) [8].

Для определения степени тяжести каждый симптом должен оцениваться пациенткой от 0 до 3 баллов: 4-21 балл соответствует легкой степени; 22-51 балл - средней степени и 52 балла и более - тяжелой степени [9].

Согласно Международной классификации болезней 10-го пересмотра (ICD-10), ПМС является самостоятельной нозологической единицей (N 94.3 Синдром предменструального напряжения). В 1998 г. в руководстве по диагностике и статистике психических расстройств, которые разрабатываются и публикуются Американской ассоциацией психи-

Таблица 1. Определение различных типов ПМС (адаптировано из N. Panay, Royal College of Obstetricians and Gynaecologists (RCOG), 2016)

\begin{tabular}{l|l}
\multicolumn{1}{c|}{ Вариант ПIМС } & \multicolumn{1}{c}{ Симпомы } \\
\hline Легкой степени & $\begin{array}{l}\text { Не нарушают течение личной/социальной } \\
\text { и профессиональной жизни }\end{array}$ \\
\hline умеренной степени & $\begin{array}{l}\text { Нарушают течение личной/социальной и } \\
\text { профессиональной жизни на оптимальном уровне }\end{array}$ \\
\hline $\begin{array}{l}\text { Тяжелой степени } \\
\text { Отказ от социальной и профессиональной } \\
\text { деятельности } \\
\text { усиление }\end{array}$ & $\begin{array}{l}\text { Неполное облегчение психологических и } \\
\text { соматических симптомов ПМС после окончания } \\
\text { менструации при наличии сопутствующей патологии }\end{array}$ \\
\hline $\begin{array}{l}\text { Предменструальное } \\
\text { дисфорическое } \\
\text { расстройство }\end{array}$ & $\begin{array}{l}\text { Критерий, предложенный Американской } \\
\text { ассоциацией психиатров }\end{array}$ \\
\hline
\end{tabular}


атров, ПМДР также было представлено в качестве отдельной нозологической единицы. ПМДР включает 11 симптомов (10 психоэмоциональных и 1 поведенческий симптом). В 2008 г. эксперты Международного общества по предменструальным расстройствам представили унифицированный вариант классификации и диагностики данного состояния, включающий основные и дополнительные симптомы [10].

Кроме того, в зависимости от преобладания тех или иных симптомов, выделяют следующие клинические формы ПМС:

- психовегетативная (раздражительность, депрессия, плаксивость, обидчивость, агрессивность, сонливость, забывчивость и т. д.);

- отечная (отечность, пастозность различных частей тела при положительном диурезе);

- цефалгическая (головная боль, приступы мигрени);

- кризовая (панические атаки, сопровождающиеся повышением артериального давления, ощущением сдавления за грудиной, похолоданием и онемением конечностей и т.д.);

- атипичная (гипертермия, офтальмоплегия, гиперсомния, циклическая аллергия и т. д.).

Уровень компенсации определяется в зависимости от прогрессирования симптомов ПМС: компенсация - симптомы ПМС с возрастом не прогрессируют и прекращаются с наступлением менопаузы; субкомпенсация - усугубление тяжести симптомов ПМС с возрастом и прекращение их с наступлением менопаузы; декомпенсация продолжение симптомов ПМС даже в течение нескольких дней после менструации и постепенное сокращение «светлых» промежутков [9].

В 2016 г. на сайте «Британского медицинского журнала» была опубликована обзорная статья, посвященная основным дифференцированным подходам коррекции ПМС (www.groupe. bmj.com) (табл. 2). В представленном обзоре Nick Panay продемонстрировал широкий взгляд на проблему ПМС с акцентом на недавние попытки глобального консенсуса установить основные дефиниции ПМС. Обзор включил в себя базы данных Королевского колледжа акушерства и гинекологии, Кохрановской библиотеки: систематические обзоры Кохрановской библиотеки, информационные сервисы DARE, EMBASE, TRIP, Medline (1966-2006), Psych INFO (1960-2006), CINHAL (1982-2006) и BMI (1985-2006).

В работе обсуждаются фармакологические и немедикаментозные методы лечения ПМС с точки зрения дока- зательной медицины (табл. 2). При наблюдении пациенток с предменструальным синдромом необходимо придерживаться индивидуального терапевтического подхода для каждой пациентки и, конечно же, не пренебрегать необходимостью коррекции образа ее жизни. Например, нет сомнений в том, что снижение стрессовых нагрузок на женщину способствует нивелированию симптомов ПМС. Кроме того, соблюдение диеты, ограничение приема алкоголя и кофеина, физические упражнения также благоприятно сказываются на течении ПМС. Женщины, в зависимости от специфичности симптомокомплекса ПМС, нуждаются в многопрофильном подходе к решению данной проблемы. И конечно, каждый специалист должен контролировать эффективность назначенной терапии по анкетамопросникам или дневникам симптомов [8].

В представленном обзоре автор в клинической практике предлагает использовать алгоритм лечения ПМС (рис.), основанный на доказательной базе эффективности и безо-

\section{Таблица 2. Основные терапевтические подходы}

\begin{tabular}{|c|c|c|}
\hline Терапия & Оценка & Примечание \\
\hline Витамин В6 & OTCIS & $\begin{array}{l}9 \text { исследований с участием } 940 \text { пациенток не представило } \\
\text { достаточных доказательств эффективности использования } \\
\text { витамина В6 в лечении ПМС }\end{array}$ \\
\hline Магний & OTC & $\begin{array}{l}\text { Только регулярное использование препаратов магния спо- } \\
\text { собствует уменьшению выраженности симптомов ПМС }\end{array}$ \\
\hline $\begin{array}{l}\text { Препараты кальция } \\
\text { и витамина Д }\end{array}$ & OTC & $\begin{array}{l}\text { Несколько исследований с небольшой выборкой предста- } \\
\text { вили повышенный риск ПМС у пациенток с дефицитом } \\
\text { витамина Д в крови и недостаточным употреблением } \\
\text { кальция в пищу. Требуются крупномасштабные клиниче- } \\
\text { ские испытания для формирования доказательной базы }\end{array}$ \\
\hline Изофлавоны & OTC & Недостаточно доказательных данных \\
\hline $\begin{array}{l}\text { Vitex Agnus castus } \\
\text { (авраамово дерево, или } \\
\text { прутняк обыкновенный) }\end{array}$ & OTC & $\begin{array}{l}\text { Недостаточно доказательных данных (но имеющиеся } \\
\text { исследования перспективны) }\end{array}$ \\
\hline $\begin{array}{l}\text { Hypericum perforatum } \\
\text { (зверобой продырявленный) }\end{array}$ & OTC & Недостаточно доказательных данных \\
\hline $\begin{array}{l}\text { Evening primrose oil } \\
\text { (масло примулы вечерней) }\end{array}$ & OTCIE & Нет достоверной разницы в сравнении с плацебо \\
\hline KOK & * & $\begin{array}{l}\text { Препараты «первой линии» терапии ПМС с доказанной } \\
\text { эффективностью }\end{array}$ \\
\hline Трансдермальные эстрогены & * & $\begin{array}{l}\text { Трансдермальные эстрогены в сочетании с гестагенами } \\
\text { эффективны для коррекции симптомов ПМС в сравнении с } \\
\text { плацебо, но не обеспечивают контрацептивного действия }\end{array}$ \\
\hline $\begin{array}{l}\text { Аналоги гонадотропин- } \\
\text { рилизинг-гормона }\end{array}$ & HS & Рекомендованы для коррекции тяжелой степени ПМС \\
\hline Прогестерон/прогестагены & $\mathrm{IE} / \mathrm{IE}$ & $\begin{array}{l}\text { Данные относительно эффективности либо отсутствуют, } \\
\text { либо противоречивы }\end{array}$ \\
\hline Гистерэктомия с придатками & HS & $\begin{array}{l}\text { Окончательный метод подавления овуляции, но в связи с } \\
\text { большим количеством осложнений в практике применяет- } \\
\text { ся достаточно редко }\end{array}$ \\
\hline СИо3С & * & $\begin{array}{l}\text { Следует рассматривать как один из вариантов первой } \\
\text { линии коррекции ПМС }\end{array}$ \\
\hline
\end{tabular}

Примечание. СИОЗС - селективные ингибиторы обратного захвата серотонина; ОТС (Over the counter medications) - лекарственное средство, назначаемое без рецепта; IS (Not recommended, insufficient evidence for safety) - не рекомендовано, нет доказательной базы в отношении безопасности; IE (Not recommended, insufficient evidence for efficacy) - не рекомендовано, нет доказательной базы в отношении эффективности; * - достаточно доказательных данных, чтобы использовать в клинической практике; HS (Recommended for hospital specialist use) - рекомендовано для использования клиницистами. 
пасности представленных терапевтических схем, фармакологическое влияние которых направлено либо на подавление функции яичников, либо селективное ингибирование обратного захвата серотонина [8]. Назначение последних является прерогативой психоневрологов или психиатров.

Вследствие вовлечения гормональных флуктуаций в патогенез развития ПМС подавление овуляций является патогенетически обоснованным. На сегодняшний день терапевтический эффект может быть достигнут при назначении даназола, аналогов гонадотропин-рилизинггормона. Однако побочные эффекты на фоне приема данной гормональной терапии могут оказаться тяжелее самого заболевания [10,11].

Интерес к комбинированным гормональным контрацептивам особенно возрос после синтеза уникального гестагенного компонента дроспиренона (ДРСП), превосходящего по выраженности своего действия прогестерон и спиронолактон. ДРСП сочетает в себе 3 эффекта, свойственные для натурального прогестерона, - прогестагенный, антиандрогенный и антиминералкортикоидный, каждый из которых вносит свой вклад в лечение ПМС. В физиологичном менструальном цикле задержка жидкости, потенцирующаяся эстрогенным компонентом комбинированного орального контрацептива (КОК), эффективно компенсируется антиминералкортикоидным действием ДРСП. Этот эффект чрезвычайно актуален в плане коррекции отечной формы ПМС, улучшения переносимости КОК. Возможность пролонгированного приема препарата основана на высокой биодоступности и отсутствии кумуляции в организме при многократном приеме $[12,13]$.

В статье представлен собственный опыт использования ДРСП-содержащих гормональных контрацептивов.

\section{Рисунок. Алгоритм лечения предменструального синдрома в зависимости от степени тяжести, предложен- ный RCOG в 2007 г.}

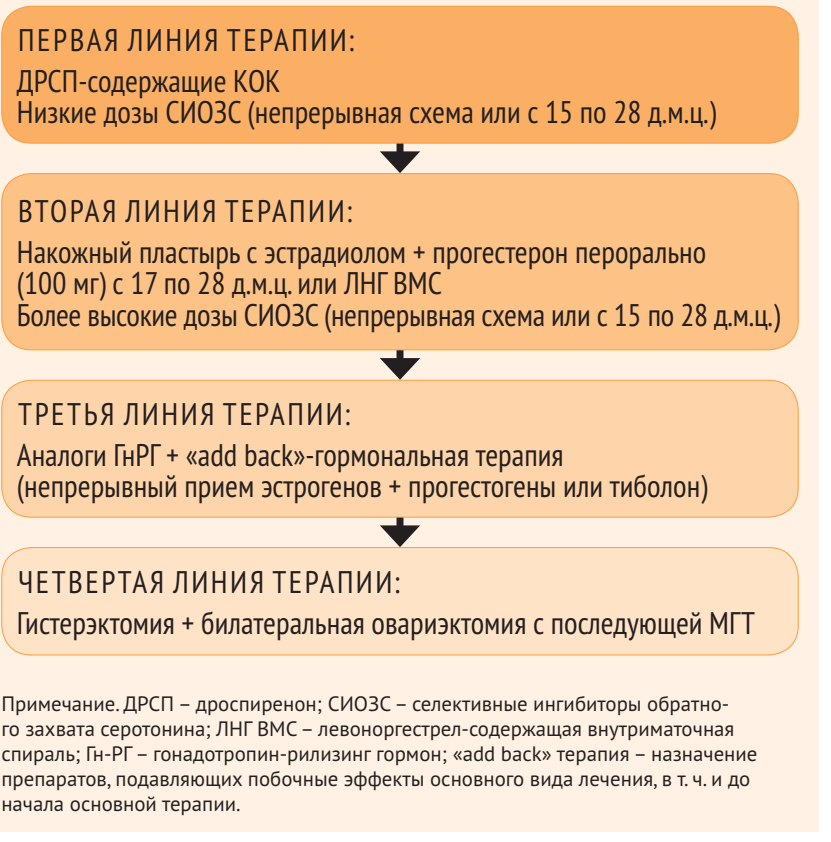

\section{Клинический случай 1.}

На прием обратилась пациентка П., 33 лет, с жалобами: - на подавленное настроение с компонентом агрессии, - чувство усиления сердиебиения,

- эмоциональную лабильность, сопровождающуюся чувством обиды,

- прибавку массы тела (со слов, на 1-2 кг) за 4-6 дней до менструации в течение 4 последних лет с заметным прогрессированием к настоящему времени.

Пациентка обратила внимание на то, что вышеуказанные симптомы ухудшают коммуникативные способности и трудоспособность. При установлении клиникоанамнестических данных у пациентки отмечалась вегетососудистая дистония по гипотоническому типу, хронический пиелонефрит в анамнезе. Наследственной патологии не выявлено. Возраст менархе - 13 лет, менструации установились сразу, по 7 дней, через 28-30 дней, умеренные, болезненные. Беременности не было. При ультразвуковом исследовании органов малого таза патологии не обнаружено, признаки произошедшей овуляции.

Пациентке было предложено в течение 2 менструальных циклов заполнять менструальный календарь, предложенный NAPS (wWw.pms.org.uk) [7], все симптомы оценивались по степени тяжести от 0 до 3 баллов. В ходе наблюдения были диагностированы такие дополнительные симптомы ПМС, как нарушение сна, повышенный апnетит, нагрубание молочных желез. При подсчете баллов определена средняя степень тяжести (24 балла), хотя при первичном приеме (без анкетирования) степень тяжести ПМС относилась к легкой категории.

Так как преобладал психологический вариант нарушений, был верифицирован диагноз: ПМС средней степени тяжести. Психовегетативная форма.

Назначена терапия КОК - (ДРСП 3 мг + этинилэстрадиол (ЭЭ) 0,03 мг) по схеме «21 + 7» на 3 месяца с обязательным ведением менструального календаря и оченкой симптомов ПМС. При повторном приеме через 1 месяи полностью купировалось агрессивное состояние, чувство усиленного сердиебиения, степень тяжести симптомов перешла в легкую, что соответствовало 16 баллам. Пациентка отмечала хорошую переносимость гормонального препарата и полное купирование болезненности во время менструации. Через 3 месяца наблюдения среди вышеперечисленных жалоб женшина отметила периодическое нарушение сна, не нарушающее трудоспособности. В связи с хорошей переносимостью и стойким положительным эффектом пациентке было предложено продолжить прием данного КОК.

\section{Клинический случай 2.}

Пациентка Н., 22 лет, с жалобами:

на головную боль распирающего характера с чувством незначительной пульсации в височной области,

раздражительность и беспокойство за 2-з дня до и в течение 2-3 дней во время менструации. Отмеченные жалобы появились 2 года назад, с постепенным усилением симптомов.

Из соматических заболеваний у пациентки диагностирован рассеянный склероз (ремиссия). В возрасте 4 лет пациентка перенесла вирусную инфекцию, осложнившуюся 
менингитом. В связи с основным заболеванием пациентку беспокоили нарушение зрения, мышечная слабость, выборочная утомляемость мыши. Cостоит на учете у невролога, получает соответствующую терапию. Наследственной патологии не выявлено. Возраст менархе - 14 лет, менструации установились сразу, по 4-5 дней, через 28-30 дней, умеренные, безболезненные. Половой жизнью не живет. При ультразвуковом исследовании органов малого таза патологии не обнаружено. На основании перечисленных жалоб, проведения анкетирования был сформулирован диагноз: ПМС средней степени тяжести (22 балла). Цефалгическая форма. Дисменорея первичная. Рассеянный склероз (ремиссия).

Лечашим неврологом была одобрена схема лечения пациентки ДРСП-содержащими гормональными контраиептивами (ДРСП 3 мг + ЭЭ 0,02 мг).

Через 1 и 3 месяца приема КОК проводилось тестирование, которое позволило диагностировать облегчение симптомов ПМС до 12 и 6 баллов соответственно. Кроме того, у пациентки полностью купировались болевые ощущения во время менструации, отмечалась стойкая ремиссия основного заболевания и заметно улучшилось качество жизни. Пащиентка продолжила прием препарата.

\section{Клинический случай 3.}

Пациенка П., 36 лет, на приеме отметила:

выраженную сонливость,

- снижение когнитивной функции за 7-8 дней до менструации, которая полностью купируется на 2-й день менструального цикла.

Пациентка была вынуждена сменить место работы в результате прогрессирования перечисленных симптомов за последние 5 лет. Из соматических заболеваний отмечает лишь хронический гастрит, аппендэктомию в детстве. Менструальная функция не нарушена. В анамнезе 2 своевременных самопроизвольных родов, без особенностей. Наследственность не отягощена. При ультразвуковом исследовании органов малого таза - интерстициальные миоматозные узлы по передней стенке матки 7 и 11 мм. На основании выявления жалоб, проведения тести- рования и обследования выставлен диагноз: ПМС тяжелой степени (декомпенсация). Гиперсомническая форма. Множественная миома матки небольших размеров. В данной ситуации при проведении тестирования и оценки тяжести симптомов в баллах ПМС относится к умеренной степени (по баллам), но в связи в прогрессированием данное состояние мы отнесли к тяжелой форме. Следует отметить, что по месту жительства врач акушер-гинеколог назначал дидрогестерон в ииклическом режиме, что привело к резкому ухудшению состояния.

Нами назначена терапия КОК (ДРСП 3 мг + ЭЭ 0,03 мг) по схеме «21 + 7» на 3 месяца с обязательным ведением менструального календаря с оценкой симптомов ПМС. Пациентка не явилась на прием через 1 месяи. Через 3 месяиа при опросе выяснилось, что вышеперечисленные жалобы полностью купировались через 1,5 месяца. Пациентка также продолжила терапию.

Наш опыт позволил определить важность проведения тестирования и оценки симптомов ПМС в баллах. Ретроспективно пациентки, как правило, предоставляют информацию о своих проблемах в меньшем объеме, и клиницист не способен полноценно оценить тяжесть заболевания и эффективность назначенной терапии. Кроме того, с помощью простого тестирования в нашей практике встречались случаи, когда у пациентов были диагностированы сопутствующие заболевания, симптоматику которых пациентки принимали за ПМС, т. к. они просто усиливались в предменструальные дни.

В заключение необходимо отметить, что на сегодняшний день проблема ПМС представлена широко и современные женщины не желают изменять свою активность в связи с их физиологическими особенностями. Ограничение физической активности и снижение качества жизни может быть напрямую ассоциировано с соматовегетативными и обменно-эндокринными проявлениями данного синдрома. Поэтому именно патогенетически обоснованное лечение с использованием ДРСП-содержащих контрацептивов позволяет достичь максимального эффекта и решить медицинскую и социальную задачу.

\section{ЛИТЕРАТУРА}

1. Сметник В.П., Тумилович Л.П. Неоперативная гинекология. М., 2005, с. 591. / Smetnik V.P., Tumilovich L.P. Non-operative gynecology. M., 2005, p. 591.

2. Буданов П.В. Лечение предменструального синдрома: современные представления и перспективы. Трудный пациент, 2012, 2-3: 18. / Budanov P.V. Premenstrual syndrome therapy: up-to-date ideas and prospects. Trudny Patsient 2012, 2-3-:18

3. Сметник В.П., Цвелев Ю.В., Кира Е.Ф. Коррекция нейроэндокринных нарушений в гинекологии. СПб, 1999. С.99. / Smetnik V.P., Tsvelev Y.V., Kira E.F. Correction of neuroendocrine disturbances in gynecology. SPb, 1999. P.99.

4. Балан В.Е., Ильина Л.М. Предменструальный синдром. Лечащий врач, 2008, 3: 55-59. / Balan V.E., Ilyina L.M. Premenstrual syndrome. Letchaschiy vrach, 2008, 3: 55-59.

5. Reid RI, Case AM. Premenstrual syndrome and menstrual-related disorders. In: Clinical repro- ductive medicine and surgery. Eds. Facone T., Hurd WW. Philadelphia (PA): Mosby Elsevier, 2007, p.335-51.

6. Кузнецова И.В., Коновалов В.А. Современная терапия предменструального синдрома. Гинекология, 2013, 3: 20-25. / Kuznetsova I.V., Konovalov V.A. Modern therapy of premenstrua syndrome. Gynekologia, 2013, 3: 20-25.

7. National Association for Premenstrual Syndrome (NAPS).Advice for sufferers and health professionals. www.pms.org.uk.

8. Panay N. Management of premenstrual syndrome. Review. May, 2016. Published by group. bmj.com.

9. Ткаченко Л.В., Курушина О.В., Атагаджиева М.С. Качество жизни женщин с предменструальным синдромом как критерий медикализации. Проблемы социальной гигиены, здравоохранения и истории медицины, 2010, 2: 14. / Tkachenko L.V., Kurushina O.V., Atagadjieva M.S. Quality of life of women with premenstrual syndrome as a criterion of medicalization. Problemy sotsialnoy gigieny, zdravookhraneniya $i$ istorii meditsiny, 2010, 2: 14.

10. Watts JF, Butt WR, Logan ER. A clinical trial using danazol for the treatment of premenstrual tension. Br J ObstetGynaecol, 1987, 94: 30-34.

11. Wyatt KM, Dimmock PW, Ismail KM, Jones PW, O'Brien PM. The effectiveness of GnRHa with and without 'add-back' therapy in treating premenstrual syndrome: a meta analysis. $\mathrm{Br} \mathrm{J}$ Obstet Gynaecol, 2004, 111: 585-593.

12. Royal College of Obstetricians and Gynaecologists (RCOG).Management of Premenstrual Syndrome (Green-top Guideline. No. 48), Panay N. (ed.). December 2007. http:// www.rcog.

13. Pearlstein TB, Bachmann GA, Zacur HA, Yonkers KA. Treatment of premenstrual dysphoric disorder with a new drospirenone-containing oral contraceptive formulation. Contraception, 2005, 72: 414-421. 